\title{
JDriver: Automatic Driver Class Generation for AFL-Based Java Fuzzing Tools
}

\author{
Zhijian Huang and Yongjun Wang * \\ College of Computer, National University of Defense Technology, Changsha 410073, China; \\ zjhuang@nudt.edu.cn \\ * Correspondence: wangyongjun@nudt.edu.cn
}

Received: 31 August 2018; Accepted: 27 September 2018; Published: 3 October 2018

\begin{abstract}
AFL (American Fuzzy Lop) is a powerful fuzzing tool that has discovered hundreds of real-world vulnerabilities. Recent efforts are seen to port AFL to a fuzzing Java program and have shown to be effective in Java testing. However, these tools require humans to write driver classes, which is not plausible for testing large-scale software. In addition, AFL generates files as input, making it limited for testing methods that process files. In this paper, we present JDriver, an automatic driver class generation framework for AFL-based fuzzing tools, which can build driver code for methods' processing files as well as ordinary methods not processing files. Our approach consists of three parts: a dependency-analysis based method to generate method sequences that are able to change the instance's status so as to exercise more paths, a knowledge assisted method to make instance for the method sequences, and an input-file oriented driver class assembling method to handle the method parameters for ordinary methods. We evaluate JDriver on commons-imaging, a widely used image library provided by the Apache organization. JDriver has successfully generated 149 helper methods which can be used to make instances for 110 classes. Moreover, 99 driver classes are built to cover 422 methods.
\end{abstract}

Keywords: driver class generation; java fuzzing; AFL; software security

\section{Introduction}

Fuzzing is an efficient and effective testing method by generating numerous inputs to reveal the vulnerabilities in the software-under-test (SUT). Recent efforts have been seen to port one of the most popular fuzzing tools AFL [1] to fuzzing Java programs. Different from binary programs, Java programs runs on Java Virtual Machine (JVM) and every public method can be tested directly with a driver class to provide basic runtime environments. Normally, the software developers write driver classes to test certain functions in the method-under-test (MUT). Their hand-written driver classes are filled with constant inputs that are only able to exercise limited paths, leaving a large part of the program not tested. AFL-based Java fuzzing tools [2,3] solve the coverage problem by making use of fuzzing techniques to generate inputs to exercise more paths. However, these tools fail to address the problem of automatic driver class generation. Both Kelinci [2] and JQF [3] rely on driver class written by testers to direct testing. This makes them not convenient for testing large-scale software. In addition, the AFL-based fuzzing tools employ files to store input, and this hinders the generation of the driver class for ordinary methods not processing files by requiring additional statements to converting the input file to correct variables. 
Our goal is to build driver classes automatically for AFL-based Java fuzzing tools. Except for providing basic runtime environments for the MUT, the generated driver class should be able to mutate the status of the class instances as well as method parameters so as to exercise all paths in the MUT with the input file generated by the fuzzing tool. The status of the class instance is decided by the fields in the class, and some class fields can only be modified through invoking methods that change the method. Thus, for each MUT, the driver class should contain method sequences to change the instance's status, statements to prepare runtime time environments for the method sequences and statements to parse the data from the input file.

We face the following challenges: the first challenge is how to build method sequences for a target method. In Java, both member fields and method parameters can affect the branch statements. The member fields declared with keyword private and protected can only be modified by member methods. We need to know what member fields are accessed by the MUT and what methods can modify them so that we can build method sequences that are able to change the status of the instance. The second challenge is how to build instances to make the method sequences work. Instances need to access member fields and invoke member methods. To instantiate classes defined in SUT as well as built-in classes properly, we need to have the knowledge of what methods can be used to create instances. The last challenge is how to handle the input file. AFL-style fuzzing tools employ file to store input, so the methods in the driver class should process the input file, and prepare basic runtime instances for the target method with data extracted from the file.

We design and implement JDriver [4], an automatic driver class generation framework for AFL-based fuzzing tools. It employs dependency analysis to build method sequences that can modify method parameters as well as the field values. It collects knowledge and uses it to instantiate classes. JDriver supports making driver classes for general methods with input-file oriented driver class assembling methods, which can handle different method parameters properly. To summarize, we make the following contributions:

(1) First study on automatic driver class generation for AFL-based Java fuzzing tools. To the best of our knowledge, we are the first to study how to make driver classes for AFL-based fuzzing tools.

(2) A novel approach to automatic driver class generation based on dependency analysis. The approach consists of a dependency analysis based method to make method sequences, a knowledge assisted method to generate class instances and an input-file oriented method to assemble driver classes.

(3) An open framework for driver class generation. We implement JDriver, an open framework that aims to support driver class generation for different purposes. Evaluation results show we are able to generate 99 driver classes containing 422 driver methods for common-imaging.

The remaining paper is organized as below: Section 2 introduces related works. Section 3 describes our approach, Section 4 depicts the implementation, and Section 5 shows our evaluation results. We illustrate our thoughts on future work in Section 6, and conclusions are given in Section 7.

\section{Related Work}

In this section, we first introduce related work on fuzzing (Section 2.1), and then we describe the the research on Java fuzzing (Section 2.2). Finally, we summarize the works on automatic unit testing (Section 2.3).

\subsection{Fuzzing}

Based on how inputs are generated, fuzzing techniques can be categorized into two types: generation-based fuzzing and mutation-based fuzzing [5]. 
Generation-based fuzzing tools generate inputs with the knowledge of the input format. These tools are easy to implement, but their effectiveness relies on the accuracy of the input model. The earliest fuzzing tool [6] generates data randomly without any knowledge of the programs, thus it is limited to test software whose input is not formatted. To test software processing formatted data, sulley [7] and peach [8] provide ways for the testers to define specification for the formatted files and network protocols. BFuzz [9] builds the knowledge of HTML and XML files into fuzzer so that it can generate valid web pages to test the browsers. Patrice et al. [10] propose using machine learning algorithms to learn the format of file format, and their method has been used to test the PDF parser of Microsoft's Edge browser. Although the techniques are evolving, it is still hard to find an effective and general way to model input for various software.

Mutation-based fuzzing tools generate inputs by mutating the existing input. These tools don't rely that much on the knowledge of input, but they suffer from low code coverage because the mutation is performed on existing inputs. AFL [1] improves this by using coverage to direct fuzzing. It instruments the SUT with codes to record the branch coverage status and mutate the input that could exercise new branches to generate new input. AFL has achieved great success in bug finding and claims to have discovered hundreds of real-world bugs [1]. A number of variants have also come out to improve AFL. In order to mutate the seed efficiently, VUzzer [11] and Steelix [12] bring in program analysis to identify "magic bytes", and use them to generate new inputs. To avoid generating inputs exercising same paths, AFLFast [13] employs a Markov-chain to direct the mutation of inputs to less-frequency branches. Angora [14] targets each branch, it solves path constraints by its efficient byte-level tainting tracking algorithm, and mutates the tainted bytes with a gradient descent based searching method to generate inputs that exercise new paths. Except for improving AFL, projects to transplant AFL to other languages and other platforms have also been built [1]. The efficiency and scalability features have won AFL a lot of research focus.

\subsection{Fuzzing Java Programs}

Although fuzzing is widely used in testing binary programs, there are fewer tools for fuzzing Java programs. jFuzz [15] is a concolic whitebox fuzzing tool based on the explicit state software model checker Java PathFinder [16]. It begins with running a program with seed input in concolic execution to resolve the path constraints, then it solves the constraint and continuously generates modified input. Like most symbolic execution tools, it suffers from path explosion, so that it is not a good choice for fuzzing large software. JFuzz [17] is another effort, which defines several mutators to mutate existing inputs and generate new inputs. It uses metamorphic relations to detect failures. However, defining metamorphic relation is difficult, and this makes it even not practical to test ordinary software, not to mention large software.

Recent efforts [2,3] have been found to port AFL to fuzzing Java codes which make use of AFL to generate inputs for the driver class written by testers. Kelinci [2] provides the Java program with AFL-style branch counting codes to track the branch exercising status of the SUT. It acts as an agent that gets input from AFL, executes the input with the Java program and transfers execution results to AFL. Similarly, JQF [3] applies AFL to fuzzing Java code in a unit testing style. Both [2] and [3] have claimed to find real-world vulnerabilities. Unfortunately, these tools require human testers to write driver classes that hinders their applications.

\subsection{Automatic Unit Testing}

Automatic unit testing aims at generating small but effective testsuites to exercise all the paths in the program. Existing approaches can be categorized into two types: random-based generation and search-based generation.

Random-based generation methods build testcases by assembling methods and assigning values randomly. These tools are simple and easy to implement, but blind, which fail to achieve high code coverage. JCrasher [18] is among the earliest efforts. It builds testcases by choosing methods from 
"parameter graph" to form method sequences and set random values for method parameters. Feedback directed random testing uses the execution information to direct future execution $[19,20]$. With the execution feedback, Randoop [19] builds inputs incrementally and classifies the inputs into redundant, illegal, contract-violating by defined contracts. In this way, the quality of the generated testcases is improved [19]. Lei et al. propose Guided Random Testing (GRT) [21], which uses program analysis to assist testcase generation. It employs static analysis to extract constants and dependency information. During the execution phase, the static information, together with dynamic analysis results, are used to create instances that may reach unexercised MUTs.

Search-based testing methods always maintain a goal, i.e., branch coverage to avoid making testcases blindly. The Search based Software Testing (SBST) Java Unit Testing Tool Contest [22] was started in 2012, which has attracted researchers to compete in this area. Evosuite [23] has been a winner for the last five contests. It employs an evolutionary algorithm to generate new testcases by merging previous testcases and minimize the size of testsuites according to the coverage metric. Sakti et al. [24] divide the test input generation problems input selecting proper methods to build class instance, calling methods to set the class under test into the proper state, and invoking target method call and searching the input space to build test input. Although these methods $[19,23,24]$ have achieved great success in the SBST contest, their abilities to reveal real-world bugs are under question [25].

\section{Approach}

\subsection{Overview}

More than providing a basic runtime environment to invoke target method and determining the execution result, AFL-based fuzzing tools require their driver class to change the instance status as well as method parameters to exercise more paths with the file generated by them. To reach this goal, we need to build method sequences that are able to change the instance status and make class instances for the method sequences with data resolved from the input file generated by the fuzzing tools.

The method sequences are designed to explore all the branches in the method under test. In Java, the branch statements may contain values derived from method parameters as well as fields. Thus, we need to get the knowledge of which fields are accessed by the method. The method sequences should contain methods to modify the accessed fields. We put forward dependency analysis based method sequences generation, which employs static analysis to extract dependency information (Section 3.2) and build method sequences according to the dependency analysis results (Section 3.3).

The method sequences require class instances to make them work. Instances can be generated from various sources: constructors and factory methods are the most common ways. However, some special classes are not easy to find proper methods to get instances, e.g., built-in classes that require additional helper methods. We put forward knowledge assisted instance generation, which builds knowledge through collecting method information in the SUT as well as the user's programming knowledge. A Helper Class is also generated to store the methods used for creating class instances (Section 3.4).

AFL-based fuzzing tools generate files to store input data. This requires our driver method to interpret the file and make instance with the interpreted data. We propose input-file oriented driver class assembling, which operates differently on methods processing files and ordinary methods not processing files. For ordinary methods, we assemble all the method parameters and make statements to recover typed values for different method parameters (Section 3.5).

\subsection{Dependency Information Extraction}

In Java, public member fields can be modified directly, while private/protected member fields can only be modified by member methods. In addition, some methods may access fields directly, while some methods access fields indirectly through invoking other methods. Thus, method calls should be taken into consideration for dependency information extraction. In our approach, we extend method 
call graph and define two directed graphs, Access Graph and Modify Graph, to store the dependency information. The vertexes are either methods or fields, and the edges in the graph are either method calls or accessing/modifying operation. Specifically, in Access Graph $A G=(V, E)$, edge from method $V_{m_{a}}$ to method $V_{m_{b}}$ indicates method $m_{a}$ invokes a call to method $m_{b}$, edge from method $V_{m_{a}}$ to field $V_{f_{x}}$ indicates method $m_{a}$ accesses the field $f_{x}$. In Modify Graph $M G=(V, E)$, the edge from field $V_{f_{x}}$ to method $V_{m_{a}}$ indicates that it is modified by method $m_{a}$, and the edge from method $V_{m_{a}}$ to method $V_{m_{b}}$ indicates that method $m_{a}$ is invoked by method $m_{b}$.

We use static analysis to extract dependency information. Algorithm 1 illustrates how Access Graph and Modify Graph are built. The algorithm begins with initializing accessGraph and modifyGraph with the methods and fields in the class (line 2-11). Afterwards, it loops over all the methods and walks over all the instructions in methods to build the graphs (line 12 to 29). If instruction inst is a method call instruction, we resolve its call target callee, and add an edge from method to callee in accessGraph. Differently, we add edge from callee to method in modifyGraph. If instruction inst is a field related instruction, we retrieve its target field. An edge from field to method is added to modifyGraph if it is a field-write instruction (lines 21-22) while an edge from method to field is added to accessGraph if it is a field-read instruction (line 24-25). In this way, both the method call relationship and the relationship between method and field are written to the two graphs.

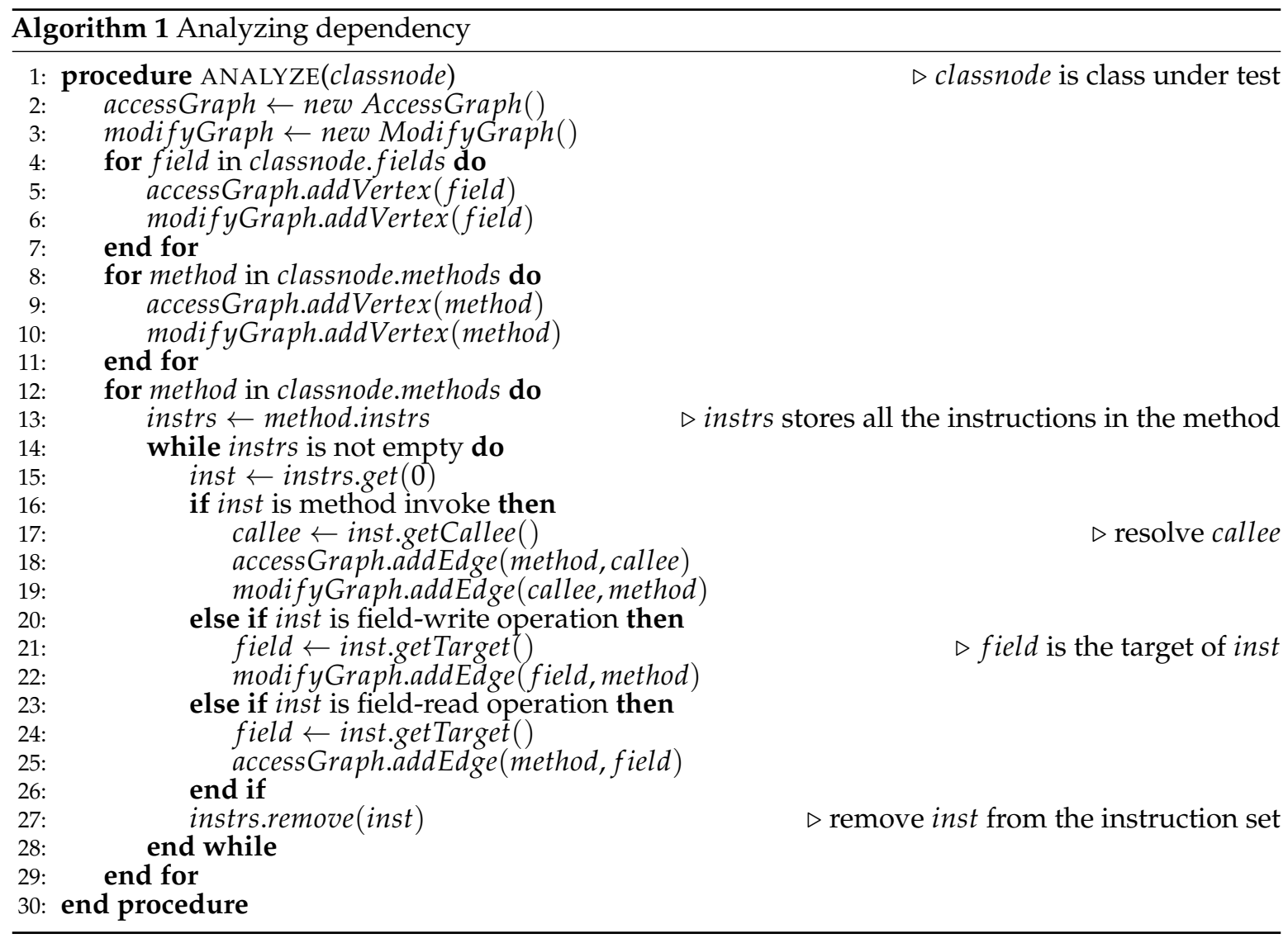

Regarding the graph theory, we come to the following two theorems:

Theorem 1. Method $m_{a}$ accesses field $f_{x}$ if and only if the two nodes $V_{m_{a}}$ and $V_{f_{x}}$ are connected in Access Graph.

Proof of Theorem 1. There are two situations in which method $m_{a}$ accesses field $f_{x}$ : direct and indirect access. In the direct situation, according to our definition of Access Graph, the direct access will be represented as an edge from $V_{m_{a}}$ to $V_{f_{x}}$ which means $V_{m_{a}}$ and $V_{f_{x}}$ are connected directly. In the indirect 
situation, method $m_{a}$ accesses field $f_{x}$ indirectly through method calls, which means that there are call sequences from method $m_{a}$ to method $m_{z}$ and method $m_{z}$ access field $f_{x}$. Method $m_{z}$ accesses field $f_{x}$ directly, so $V_{m_{z}}$ and $V_{f_{x}}$ are connected (1). The call sequences from $m_{a}$ to $m_{z}$ indicate that there is a path from $V_{m_{a}}$ to $V_{m_{z}}$ which indicates that $V_{m_{a}}$ and $V_{m_{z}}$ are connected (2). Combining (1) and (2), $V_{m_{a}}$ and $V_{f_{x}}$ are connected. Thus, if method $m_{a}$ accesses field $f_{x}$, the two nodes are connected in the Access Graph. Reversely, if $V_{m_{a}}$ and $V_{f_{x}}$ are connected, there is a path between $V_{m_{a}}$ and $V_{f_{x}}$. If the length of the path is 1 , it means the method $m_{a}$ access field $f_{x}$ directly. If the length is bigger than 1 , there are more than two vertexes in the path, namely $V_{m_{a}}, V_{m_{b}} \ldots V_{m_{z}}, V_{f_{x}}$. The vertex $V_{m_{z}}$ and $V_{f_{x}}$ are connected directly meaning method $m_{z}$ access field $f_{x}$ directly (3). The path from $V_{m_{a}}$ to $V_{m_{z}}$ indicates that there are call sequences from method $m_{a}$ to $m_{z}(4)$. Combining (3) and (4), we get that method $m_{a}$ accesses field $f_{x}$ indirectly. Thus, if $V_{m_{a}}$ and $V_{f_{x}}$ are connected, method $m_{a}$ access field $f_{x}$.

Theorem 2. Method $V_{m_{a}}$ can modify field $V_{f_{x}}$ if and only if the two nodes are connected in Modify Graph.

Theorem 2 can be proved in the same convention of Theorem 1 . Theorem 1 explains how we can find the member fields accessed by the given target. While Theorem 2 provides us with a way to find the member method that can modify target member fields. We use AccesssSet ${ }_{m}$ to represent the set of fields that are accessed by method $\mathrm{m}$ and ModifySet $f$ to be the set of methods that can modify field $\mathrm{f}$. Actually, AccesssSet $m$ is made up of all the field nodes that are connected with the specified method $m$ and ModifySet $f$ is made up of all the method nodes that are connected with field $f$.

\subsection{Method Sequence Building}

The method sequences are used to modify the status of the instance. Apart from invoking methods to change target fields, the public fields can also be changed by assigning values directly. Thus, we extend method sequences to include field to indicate that the field can be modified directly.

We build method sequences on dependency information. For the MUT, we can get its accessed fields set with Access Graph, and the Modify Graph assists us with retrieving a set of methods that can modify the target field. Algorithm 2 illustrates how we build method sequences with Access Graph and Modify Graph. An empty array ms is initialized to store method sequences. For the given method mut, we first resolve the mut's accessSet accessSet $_{m u t}$ (line 2). Then, we build the method sequence incrementally by iterating over the accessSet $_{m u t}$ (line 4-10). For every field in the accessSet $_{m u t}$,

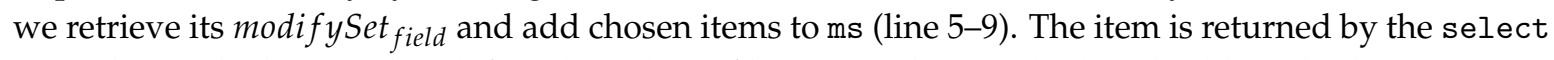
procedure, which is used to define the policy of how we select methods to build method sequences.

Policy to select method. As static analysis is conservative, the extract dependency information may not be accurate. Thus, we need to implement different policies to get better performance. In our implementation, we design a policy to prioritize the field item and select the method whose method parameters are simplest to make. In the procedure select, we first check if the field is public and its type is primitive. If it is, we return it directly. If not, we examine whether the existing methods in the method sequences can modify the target field (lines 16-20). If such methods exist, null is returned to avoid duplicate modification. If not, we sort the method in the modifySet $_{\text {field }}$ (line 21) and return the first method (line 22). In our case, the methods are sorted by the simpleness of method parameters, which is measured by the number of primitive parameters in the method.

\subsection{Knowledge Assisted Instance Generation}

Normally, instances are created by the constructor of the specified class. In addition, factory methods that make instance as its return value can also be used to generate instances. However, for built-in classes provided by The Java Platform, Standard Edition (Java SE), e.g., String, it is not easy to find proper constructors or factory methods, they require additional methods to make instances. We name these methods to create instances as knowledge. Our knowledge assisted instance generation method builds knowledge through collecting related methods for the SUT as well as making methods 
from the users' knowledge. As instance are frequently used in driver class, we build a Helper Class to store all the instance generation methods.

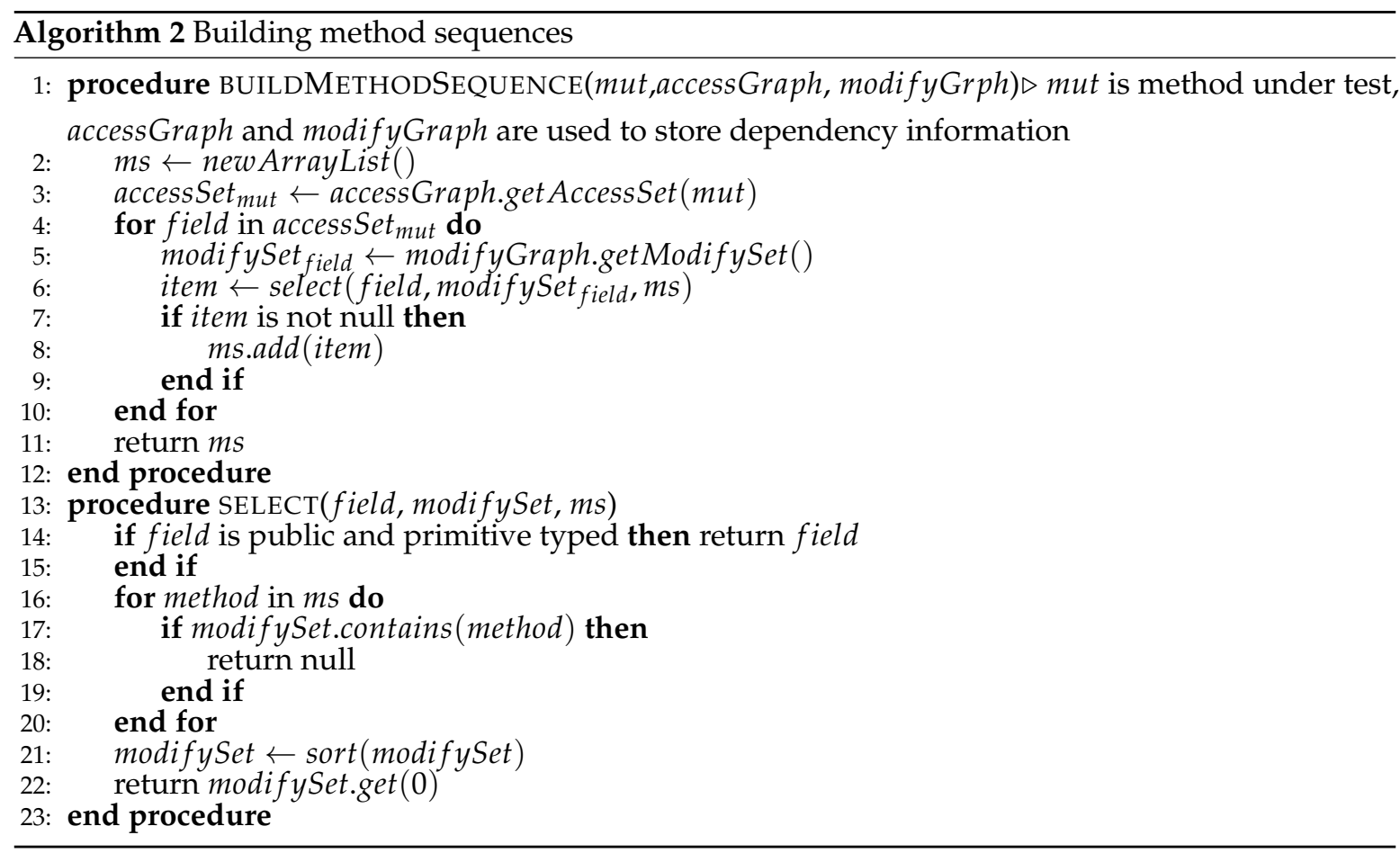

Collecting instance generation methods. We define a type table to store factory methods and class constructors for the SUT. The type table uses class type as the key, and the value of the key is a set of methods. We build type table by walking all the methods in the SUT as illustrated in Algorithm 3. For each method in the SUT, we first resolve its return type returnType (line 5). Afterwards, we decide if returnType has already existed in the type table. If it has, we add it to the corresponding method set (line 7). If it has not, we create a new set, and put it into the new set, and add an item to the type table (lines 9-11). Apart from SUT, we also build type table for its dependent libraries.

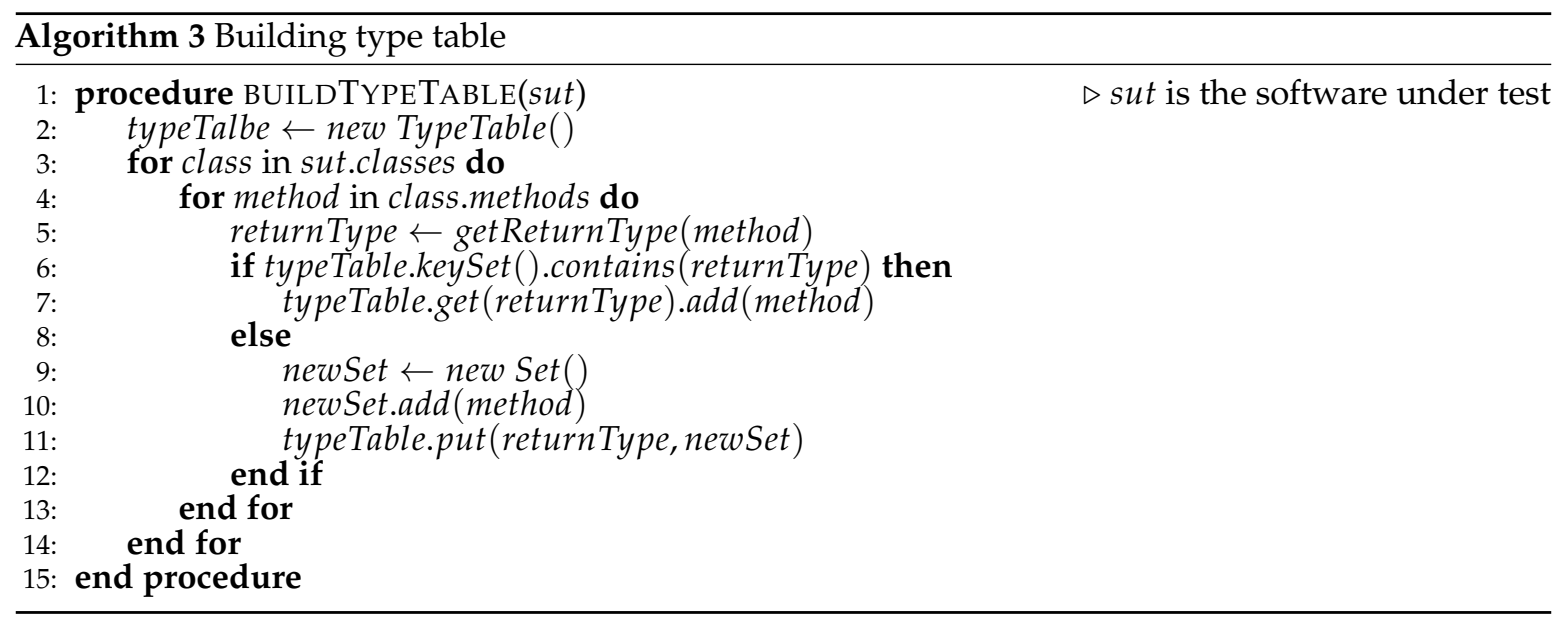

Knowledge for built-in classes. We add knowledge for classes provided by the Java Platform. Specifically, we cover most of the classes defined in the java.util package, which contains the container classes such as Set, the java. lang package, which defines classes that are fundamental to the design of the Java programming language such as String, and the classes in java.io package, which contains classes to handle system input/output [26]. Figure 1 shows two sample knowledge 
methods. Method get_String returns a String instance which comes from the input parameter arg0. Method get_File returns a File instance, which is created by the new expression with the method parameterarg0.

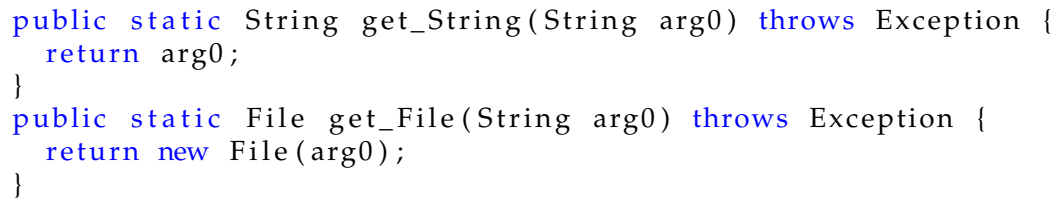

Figure 1. Sample knowledge method.

Building Instance Helper Class. Instantiating class instances are frequently used during testing. To avoid generating it repeatedly, we build an Instance Helper Class to handle the generation of instances. Algorithm 4 shows the building process for Instance Helper. It starts with initializing typeSet to include all the buildable classes (lines 2-7). Afterwards, it builds instance helper methods with buildInstanceHelper (line 8 ). When the processing finishes, InstanceHelperClasses assembles all the methods, and adds miscellaneous codes to build a compilable Helper Class (line 9). In the buildInstanceHelper method, it initializes unprocessed as a copy of typeSet, and then it walks over all the types in typeSet to build helper methods (lines 13-18) with buildType. Procedure buildType builds helper methods for each type and returns the number of generated helper methods. If buildType builds more than one helper method successfully, then the type is removed from unprocessed (line 16). Since some class constructors rely on other classes, it is necessary to build helper class recursively to cover these classes (lines 19-20). In buildType, it firstly resolves the methodSet for the given type type. Then, it iterates over all the methods in the methodSet to build helper method (lines 28-34). Each time a helper method is generated, it is appended to InstanceHelperClasses. Note that, in order to simplify the method inputs, our helper method only employs primitives or String as method parameters.

\subsection{Input-file Oriented Driver Class Assembling}

For a class-under-test (CUT), we build driver method separately for each public method and assemble the driver methods into a driver class. AFL-based fuzzing tools generate files to store the input data. If the MUT processes files directly, we can pass the file directly as a method parameter. However, in most cases, the methods don't do so. For these methods, their driver methods need to process the input file and present the data to make variables for the methods. Our input-file oriented driver class assembling method works differently on ordinary methods not processing file and methods processing file.

Testing if method processes file. As file processing methods use built-in classes such as File to handle files, we design the following heuristic to determine whether the method processes files directly: (1) the method parameters contain file related class instances such as File; and (2) there is a String typed method parameter which flows to a file opening method.

Ordinary methods not processing files. Our driver method starts with extracting the input file to a byte array, and then it resolves the values for the method parameters sequentially from the byte array. Algorithm 5 shows the building process. Method makeStatements begins with declaring a variable position to mark the position in the byte array, and it iterates over the items in the method sequences to make statements. If the item is a field, it makes an assigning statement directly with makeField (lines 35-40). If it is a method, it makes statements to declare variables as well as the statement to invoke the method with makeMethod (lines 11-34). For each method parameter, method makeMethod applies different rules according to their types. There are two categories of types in Java: primitive types and reference types. (1) Primitive types. Primitive typed data has fixed sizes, and we can make it directly from the input bytes. Method makeVariableStatement generates statements like 
this: int $\mathrm{a}=\mathrm{Helper}$.getInt (inputs, position). (2) Reference types. Class types and array types are two reference types. For class types, we firstly get a helper method from InstanceHelperClasses (line 18). If helperMethod is not null, we make statements for the helper method (line 20). For array types, if it is primitive array, we resolve its element type (line 25), and its array length (line 26). If the length is not specified, we will use a random number to replace it. We make statements with its element type etype (line 27). If we can't get a proper helperMethod or the array is not a primitive array, we make statements with built-in knowledge (lines 21,28). Method makeMethod ends with making statements to invoke the method (line 32), and returning the position to avoid retrieving bytes from the same position (line 33). Method makeField works similarly as the primitive type in makeMethod.

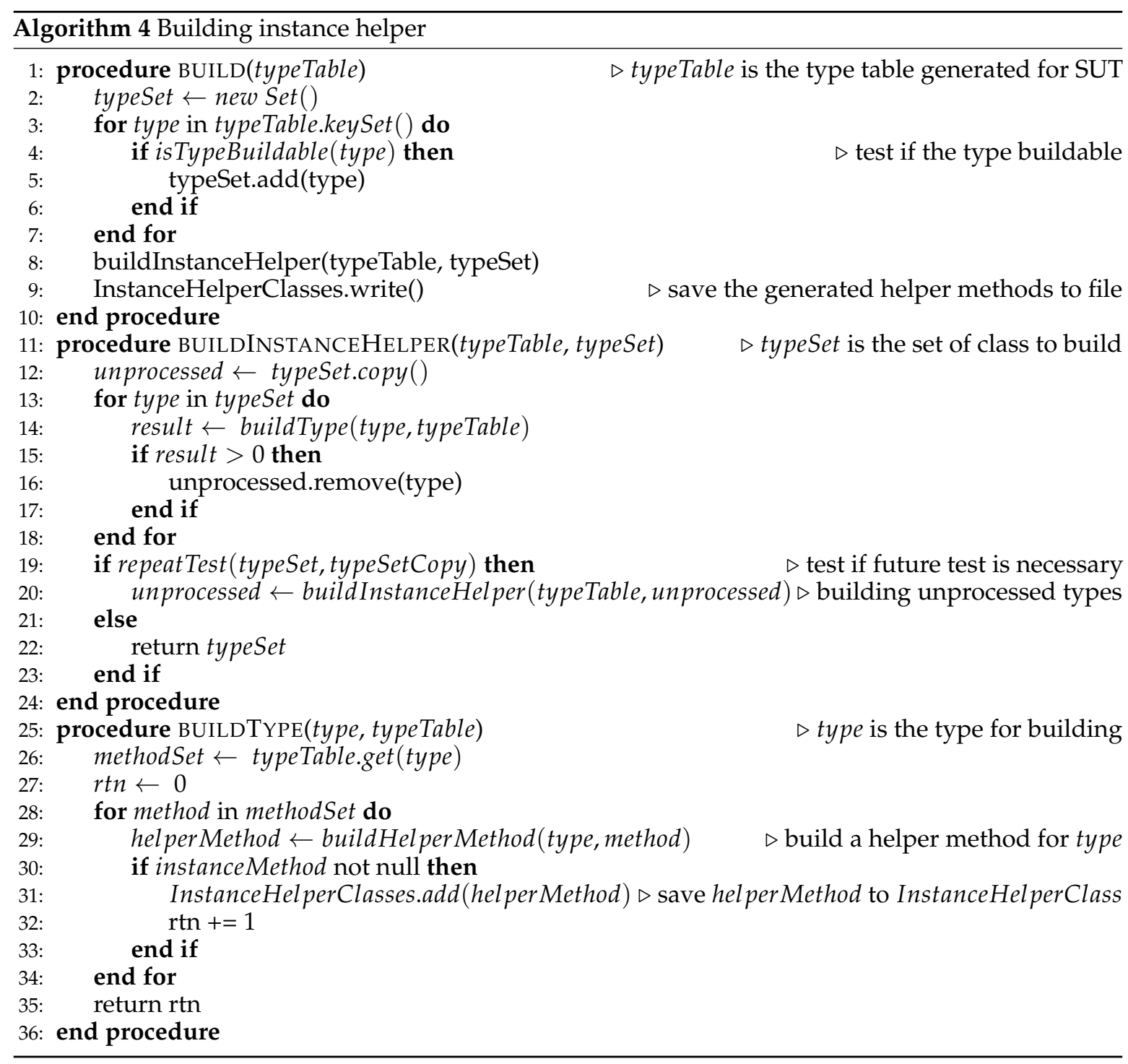

Method processing files. For these methods, we identify which method parameter is used to specify the filename, and then present the file path to the method directly. If there exist other method parameters, we use random generators to generate values. 


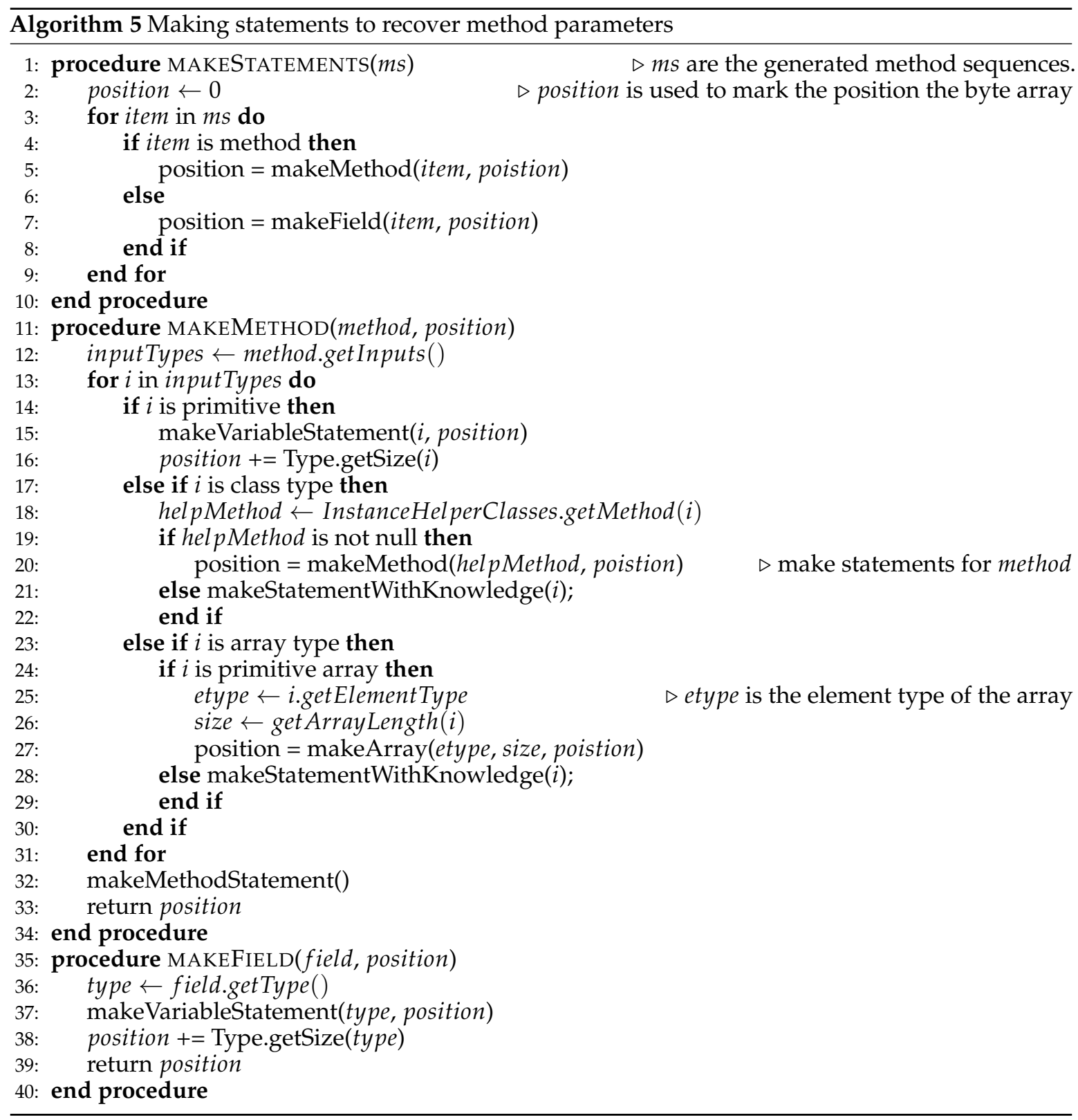

\section{Implementation}

Our automatic driver class generation approach has been implemented into JDriver with around 5000 lines of Java code. It uses the general purpose Java bytecode analysis framework ASM [27] to perform dependency analysis as well as extract class information. JDriver takes Java program as input, and produces driver classes in the following steps:

(1) preprocessing. JDriver starts with analyzing the SUT to collect methods used for instance generating and analyzing the attributes of the classes.

(2) extracting dependency information. For each CUT, we extract dependency information, Access Graph and Modify Graph according to the algorithm described in Section 3.2.

(3) building method sequence. For each MUT, we resolve its accessSet $_{m u t}$ and build method sequences that are able to change the values in the access Set $_{m u t}$.

(4) building instance helper class for instance generation. For the SUT, we build knowledge by collecting constructors and factory methods in the SUT. For built-in classes, we design additional helper methods. With the knowledge, we produce a Helper Class to save methods for class instantiating. 
(5) assembling driver class. Finally, with the method sequences and instance generation helper methods, we build statements to operate on the input file and declare variables for the method sequences.

\section{Evaluation}

We first demonstrate our driver class generation with a simple example in Section 5.1. Then, we work on the widely used image processing library commons-imaging [28] to evaluate instance generation (Section 5.2) and driver class generation (Section 5.3).

\subsection{Simple Example}

The example CUT. We use the simple example to illustrate how our approach works. Figure 2 shows the source code of the CUT. The class AClass has three member fields: $a, b$ and c. Fields a and $\mathrm{c}$ are public while $\mathrm{b}$ is private which can only be modified through public method setB (lines 15-18). In method foo (lines 18-25), both field $a$ and $b$ are involved in the branch statement in line 19. To exercise all the input space, the driver code should be able to change the values of fields a and $\mathrm{b}$ as well the method parameter $\mathrm{x}$.

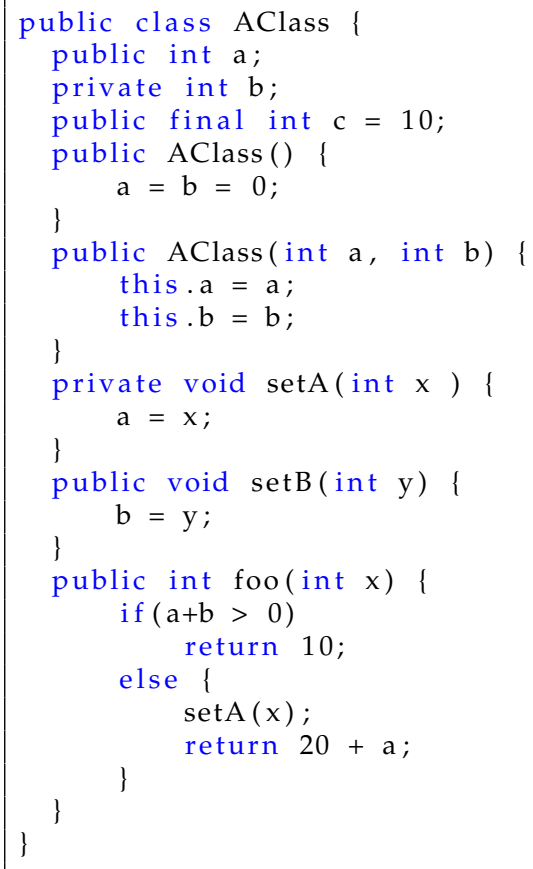

Figure 2. Sample class under test.

The driver class. As shown in Figure A1, JDriver generates a driver class AClassTest for the CUT which is made up the main method to control the testing and driver method foo_test to test method foo. The entry method main (lines 43-48) employs an array of String as input and args [0] is set to be the filename of the input. Method foo_test (lines 13-42) takes a String to represent filename. The file is extracted to a byte array data (lines 14-18), which is divided into fields and inputs array for building instances for method sequences and inputs of the target method separately (lines 19-20). Afterwards, the parameters for building AClass instance are prepared (lines 25-28), and an instance cut is generated (line 29) by the constructor. For public member field a, we modify it through assigning operation (line 30). The method parameter is recovered in lines 34-35, and the target method foo is tested in try-catch block to catch runtime exceptions (lines 37-41). Note that the generated method sequence for foo are lines 29 and 30. Our method sequences contain the statement (line 30) to change a, but don't contain separate statements to change $b$. This is because we implement a selecting method policy to ignore the fields that are changed by the constructor if the fields could not be assigned values directly. 


\subsection{Evaluating Instance Generation}

JDriver builds a Helper Class named InstanceHelper.java to save the helper methods for instance generation. JDriver successfully generates 149 helper methods for 110 classes. Figure 3 illustrates the Instance Helper Method for class ByteSourceFile, whose constructor uses a File object. In line 1, a File instance is generated by the helper method get_File, which makes a File instance from a String instance.

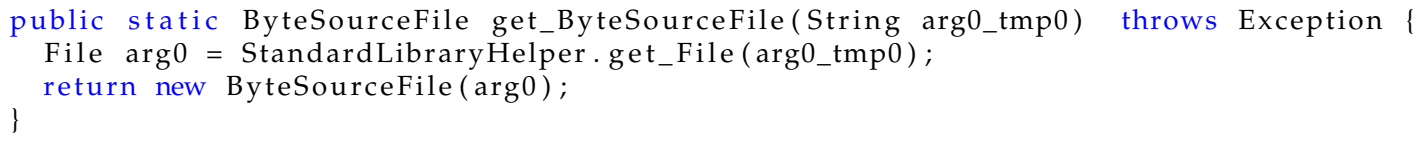

Figure 3. Sample instance helper method.

\subsection{Evaluating Driver Class Generation}

JDriver successfully builds 99 driver classes for 422 methods, 60 of which are methods processing file. Figure 4 shows the driver method generated for method convertYCbCrtoRGB, which takes three integers as the method parameter. Our dependency analysis detects there are no fields involved in the convertYCbCrtoRGB, so we only need declare variables for method parameters. Similar to the driver class in Figure A1, our driver method begins with reading data in the file and forming the byte array data (lines 2-5). The input array is later resolved to make variables (line 8). Afterwards, we declare three int variables and assign them with the values resolved from the inputs (lines 13-19). To avoid naming conflicts, each variable is named after the name of its method. These variables are later used as parameters to the convertYCbCrtoRGB. In this way, the input file generated by the AFL-based fuzzing tool is converted to method parameter of ordinary methods. The more input files it generates, the higher possibility for the driver method to exercise all paths.

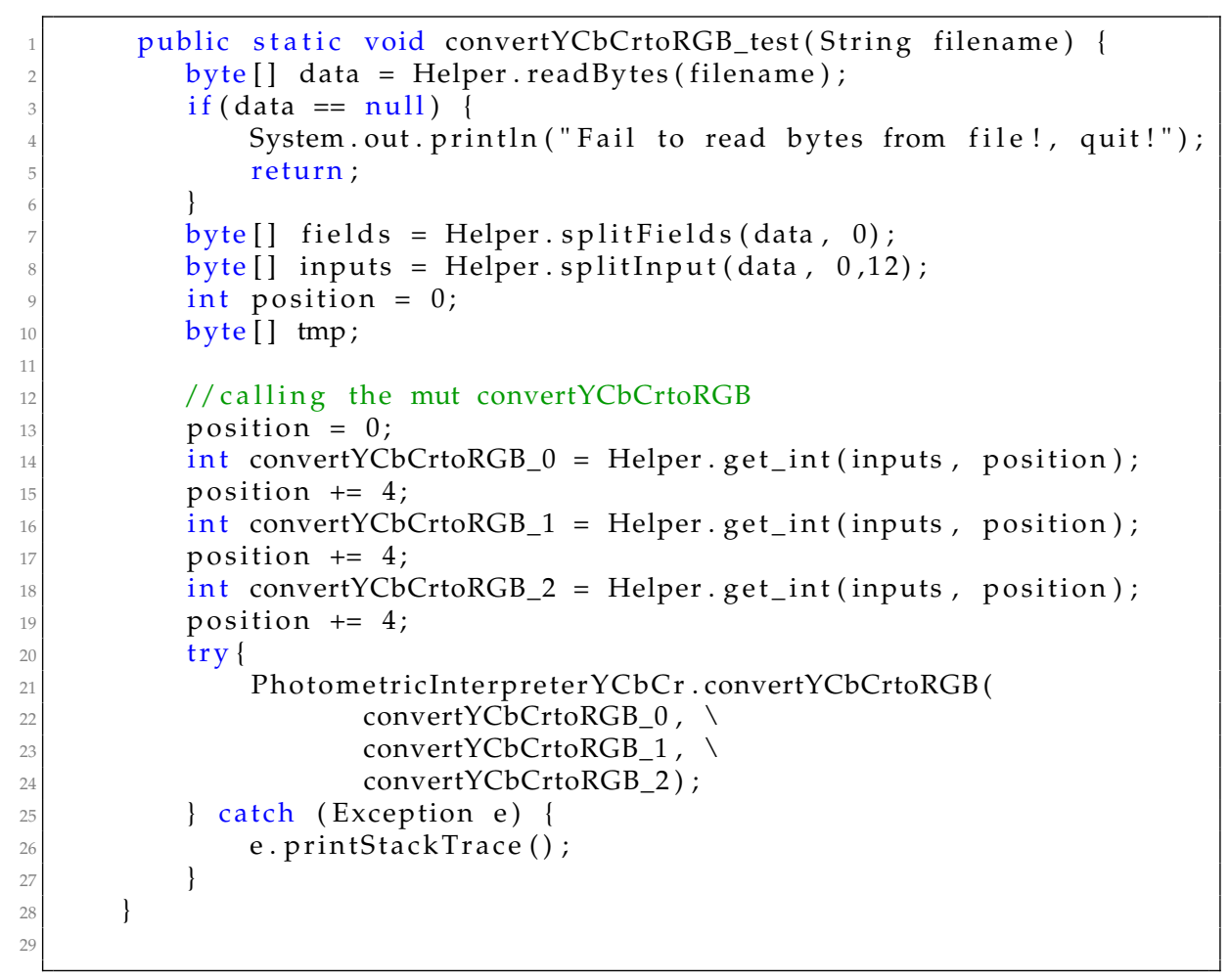

Figure 4. Sample driver method for the processing file method. 


\section{Discussion and Future Work}

Instance Generation. Although JDriver has generated hundreds of driver methods for commons-imaging, it fails to make correct instances for the following classes: (1) interface classes. Class ImageFormat is an interface, which should be initialized through classes that has implemented this interface. However, JDriver has no knowledge for generating class instance for interface, thus it fails on interface classes. (2) classes containing types not covered in knowledge base. The constructor of ByteSourceArray has String and byte [] as method parameters, but we missed the byte[] type in our helper class. This makes JDriver fail to generate instance for class ByteSourceArray. (3) classes whose constructor involves multiple String objects. The constructor method of class ByteSourceInputStream takes an InputStream and a String typed parameters. Our algorithm detects that the helper method get_InputStream can be used to make instance for class InputStream and method get_String can be used to make String instance. However, it skips the methods that use multiple String objects because the driver code only has one String object as input, so it fails to make ByteSourceInputStream instances. In addition, some instances we make are meaningless. For example, method getBufferedImage in class verb I JpegImageParser I accepts a HashMap instance and uses it to store items. Normally, we need to initialize an non-empty HashMap. However, if we assign a null to that parameter, it may continue the execution but won't reach our target branch. To summarize, we need more smart knowledge to build correct instance. In the future, we will continue working on: covering advanced Java features like subclassing, interfaces into knowledge base, covering more built-in classes, restructuring helper methods, and learning knowledge from the source code of the SUT.

Fuzzing Scheduling. Actually, fuzzing every method in Java programs is neither plausible nor necessary. (1) indirectly accessible methods should be skipped. Java puts access control attributes in every method, and methods declared with public and private can't be accessed directly. In addition, abstract classes can't be instantiated. These codes should be skipped. (2) methods that don't produce errors should be excluded. In Java, some methods are used to do simple work, they have single branches and never throw exceptions. For example, the getter methods only contain a single statement to return a field value; they can never produce exceptions. (3) methods that have been exercised are not necessary to test alone. Some methods are repeatedly implemented when fuzzing other methods; testing these methods is useless and wastes a lot of time. A proper way to schedule methods for fuzzing is to use program analysis techniques to identify the methods listed above. Static analysis can help identify the methods that have no branches and won't produce exceptions. Dynamic analysis techniques could track the execution of the methods and find out what methods have already been used.

\section{Conclusions}

Building sound driver classes for AFL-based Java fuzzing tools can improve their fuzzing ability and efficiency. In this paper, we study the automatic driver class generation problem for these tools. Our automatic driver class generation method employs dependency analysis to analyze what fields are accessed by the method, and then construct method sequences to mutate the values of the accessed fields so that the generated method sequences are able to mutate the status of the class instances. We design a knowledge assisted instance generation method to make instances for classes from various sources. In order to allow fuzzing for ordinary methods, our input-file oriented driver class generation method rebuilds method interfaces and generates statements to declare variables for parameters used in the method sequences. We implement our approach in JDriver. To the best of our knowledge, we are the first to study this problem, and JDriver is the earliest automatic driver class generation framework for AFL-based Java fuzzing tools. We evaluate JDriver on real-world library commons-imaging, and it has successfully generated 99 driver classes to cover 422 methods; this proves that JDriver is effective in driver class generation for AFL-based fuzzing tools. 
Author Contributions: Methodology, Software, Writing, Z.H.; Methodology, Supervision, Review \& Editing, Y.W. Funding: This research is supported by NSFC No.61472439, the National Natural Science Foundation of China. Acknowledgments: We'd like to express our appreciation to anonymous editors.

Conflicts of Interest: The authors declare no conflict of interest.

\section{Appendix A. The Generated Sample Driver Class}

Figure A1 illustrates the driver class we generated for sample class in Figure 2.

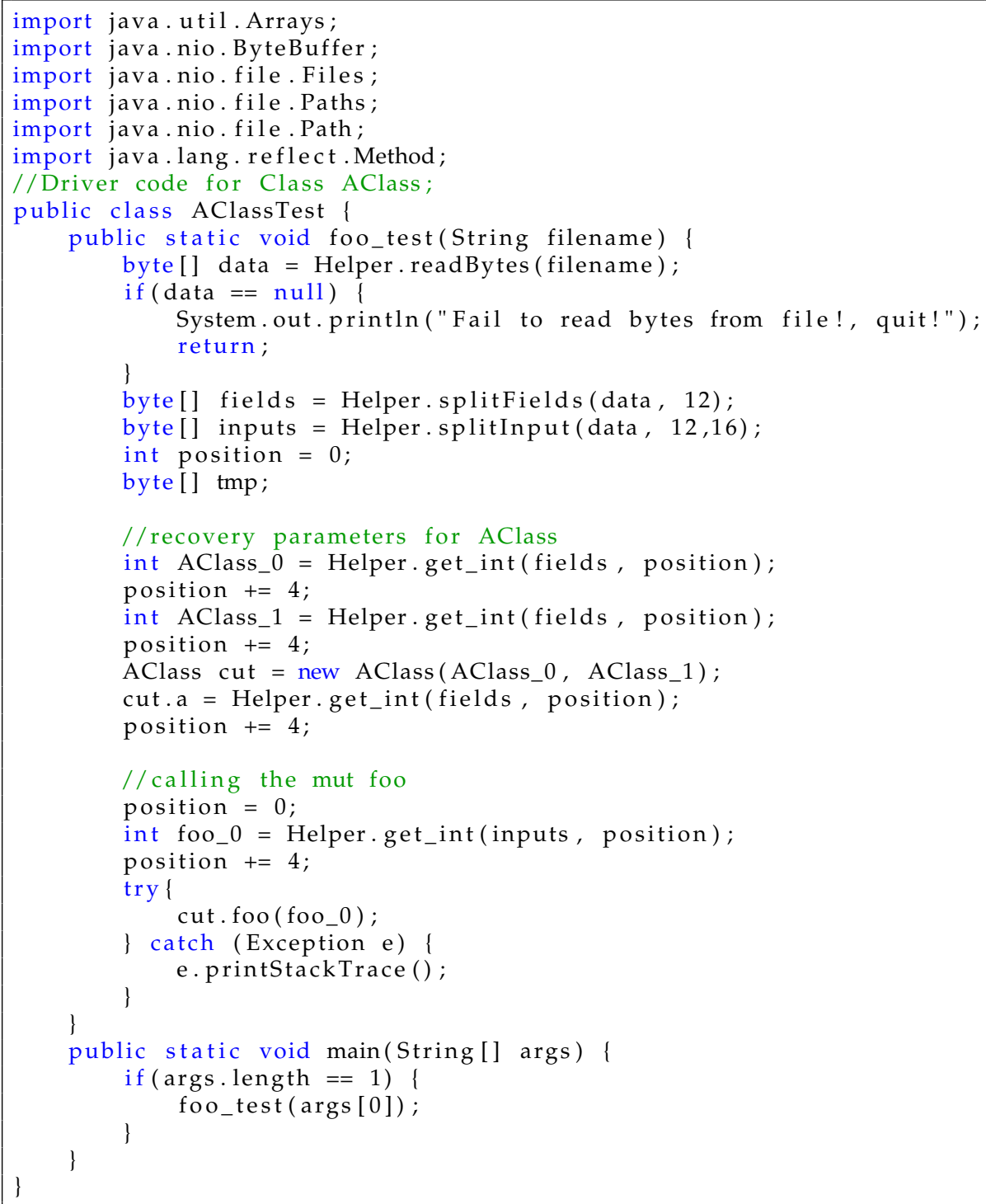

Figure A1. Driver class for class defined in Figure 2.

\section{References}

1. American Fuzzy Lop. Available online: http://lcamtuf.coredump.cx/afl/ (accessed on 21 August 2018).

2. Kirsten, R.; Luckow, K.; Păsăreanu, C.S. POSTER: AFL-based Fuzzing for Java with Kelinci. In Proceedings of the 2017 ACM SIGSAC Conference on Computer and Communications Security, Dallas, TX, USA, 30 October-3 November 2017; ACM: New York, NY, USA, 2017; pp. 2511-2513. [CrossRef]

3. JQF. Available online: https://github.com/rohanpadhye/jqf (accessed on 21 August 2018).

4. JDriver. Available online: https://github.com/qorost/jdriver.git (accessed on 1 October 2018). 
5. Li, J.; Zhao, B.; Zhang, C. Fuzzing: A survey. Cybersecurity 2018, 1, 6. [CrossRef]

6. Miller, B.P.; Fredriksen, L.; So, B. An Empirical Study of the Reliability of UNIX Utilities. Commun. ACM 1990, 33, 32-44. [CrossRef]

7. The Sulley Fuzzer. Available online: https://github.com/OpenRCE/sulley (accessed on 21 September 2018).

8. The Peach Platform. Available online: https://www.peach.tech/products/peach-fuzzer/peach-platform/ (accessed on 21 September 2018).

9. The BFuzz Platform. Available online: https://github.com/RootUp/BFuzz (accessed on 21 September 2018).

10. Godefroid, P.; Peleg, H.; Singh, R. Learn\&fuzz: Machine learning for input fuzzing. In Proceedings of the 32nd IEEE/ ACM International Conference on Automated Software Engineering, Urbana-Champaign, IL, USA, 30 October-3 November 2017; IEEE Press: Piscataway, NJ, USA, 2017; pp. 50-59.

11. Rawat, S.; Jain, V.; Kumar, A.; Cojocar, L.; Giuffrida, C.; Bos, H. Vuzzer: Application-aware evolutionary fuzzing. In Proceedings of the Network and Distributed System Security Symposium (NDSS), San Diego, CA, USA, 26 February-1 March 2017.

12. Li, Y.; Chen, B.; Chandramohan, M.; Lin, S.W.; Liu, Y.; Tiu, A. Steelix: Program-state based binary fuzzing. In Proceedings of the 2017 11th Joint Meeting on Foundations of Software Engineering, Paderborn, Germany, 4-8 September 2017; ACM: New York, NY, USA, 2017; pp. 627-637.

13. Böhme, M.; Pham, V.T.; Roychoudhury, A. Coverage-based greybox fuzzing as markov chain. IEEE Trans. Softw. Eng. 2017. [CrossRef]

14. Chen, P.; Chen, H. Angora: Efficient Fuzzing by Principled Search. In Proceedings of the 2018 IEEE Symposium on Security and Privacy, San Francisco, CA, USA, 21-23 May 2018; pp. 711-725. [CrossRef]

15. Jayaraman, K.; Harvison, D.; Ganesh, V.; Kiezun, A. jFuzz: A Concolic Whitebox Fuzzer for Java. In Proceedings of the First NASA Formal Methods Symposium, Moffett Field, CA, USA, 6-8 April 2009; pp. 121-125.

16. NASA Java PathFinder. Available online: http:/ /javapathfinder.sourceforge.net (accessed on 21 August 2018).

17. Zhu, H. JFuzz: A Tool for Automated Java Unit Testing Based on Data Mutation and Metamorphic Testing Methods. In Proceedings of the 2nd International Conference on Trustworthy Systems and Their Applications, Hualien, Taiwan, 8-9 July 2015; pp. 8-15.

18. Csallner, C.; Smaragdakis, Y. J. Crasher: An automatic robustness tester for Java. Softw. Pract. Exp. 2004, 34, 1025-1050. [CrossRef]

19. Pacheco, C.; Ernst, M.D. Randoop: Feedback-directed Random Testing for Java. In Proceedings of the Companion to the 22Nd ACM SIGPLAN Conference on Object-oriented Programming Systems and Applications Companion, Montreal, QC, Canada, 21-25 October 2007; ACM: New York, NY, USA, 2007; pp. 815-816. [CrossRef]

20. Godefroid, P.; Klarlund, N.; Sen, K. DART: Directed Automated Random Testing. In Proceedings of the 2005 ACM SIGPLAN Conference on Programming Language Design and Implementation, Chicago, IL, USA, 12-15 June 2005; ACM: New York, NY, USA, 2005; pp. 213-223. [CrossRef]

21. Ma, L.; Artho, C.; Zhang, C.; Sato, H.; Gmeiner, J.; Ramler, R. Grt: Program-analysis-guided random testing $(\mathrm{t})$. In Proceedings of the 2015 30th IEEE/ACM International Conference on Automated Software Engineering (ASE), Lincoln, NE, USA, 9-13 November 2015; pp. 212-223.

22. 10th International Workshop on Search-Based Software Testing. Available online: http:/ /sbst2017.lafhis.dc. uba.ar (accessed on 21 August 2018).

23. Fraser, G.; Arcuri, A. EvoSuite: Automatic Test Suite Generation for Object-oriented Software. In Proceedings of the 19th ACM SIGSOFT Symposium and the 13th European Conference on Foundations of Software Engineering, Szeged, Hungary, 5-9 September 2011; ACM: New York, NY, USA, 2011; pp. 416-419. [CrossRef]

24. Sakti, A.; Pesant, G.; Guéhéneuc, Y.G. Instance generator and problem representation to improve object oriented code coverage. IEEE Trans. Softw. Eng. 2015, 41, 294-313. [CrossRef]

25. Shamshiri, S.; Rojas, M.; Fraser, G.; Mcminn, P.; Arcuri, A. Do Automatically Generated Unit Tests Find Real Faults? An Empirical Study of Effectiveness and Challenges. In Proceedings of the 2015 30th IEEE/ACM International Conference on Automated Software Engineering (ASE), Lincoln, NE, USA, 9-13 November 2015.

26. Java Platform, Standard Edition \& Java Development Kit Version 9 API Specification. Available online: https: / /docs.oracle.com/javase/9/docs/api/index.html?overview-summary.html (accessed on 21 August 2018). 
27. ASM. Available online: http:/ /https:/ / asm.ow2.io (accessed on 21 August 2018).

28. commons-imaging. Available online: https://commons.apache.org/proper/commons-imaging (accessed on 21 August 2018). 